\title{
la séismicité induite par les lacs réservoirs dans son contexte géologique
}

\author{
par \\ P. Gevin \\ Professeur à I'Université Claude Bernard \\ de Lyon, France
}

\section{Introduction}

Classiquement un séisme se perçoit comme un ébranlement naturel du sol, caractérisé par sa brièveté et sa soudaineté, presque toujours accompagné de phénomènes sonores. Adoptant l'image très simplifiée qu'en donne J. Coulomb [1] on admettra que, des contraintes non hydrostatiques croissant quelque part dans l'écorce terrestre, à un certain moment, les efforts dépassent la résistance du matériau concerné et celui-ci se rompt, en un point d'abord (le foyer) puis la félure se propage dans le plan de faille; simultanément sont engendrées des ondes élastiques (séismiques) qui vont ébranler les alentours et constituent le tremblement de terre.

Une rupture, ou faille en termes géologiques, est donc nécessairement à l'origine d'un séisme, et la taille de la première est assortie à l'importance du second, tout au long de l'échelle des grandeurs. On notera toutefois que les séismes perçus directement par les hommes sont issus de foyers généralement peu profonds $(50 \mathrm{~km})$ et mettent en jeu des failles d'échelle au moins métrique, voire multikilométrique dans les grands séismes. La conservation du sens du premier mouvement transporté par les ondes premières tout au long de leur parcours intérieur à la terre, représente un fait très remarquable utilisé dans la détermination à distance du mécanisme focal, possible quand on dispose d'un nombre suffisant d'observatoires bien placés.

Un autre fait non moins important est que les épicentres (projection des foyers à la surface) se localisent systématiquement dans de longues bandes découpant la terre en "plaques" ayant forme de calottes sphériques. Les limites des plaques sont par définition zones de mouvement et si elles sont bien allongées à l'échelle de la terre elles ont parfois une largeur supérieure à $2000 \mathrm{~km}$. C'est ainsi que la zone dite alpine dans sa partie occidentale par exemple, s'étend du $30^{\circ}$ au $53^{\circ}$ parallèle.

Les mouvements se groupent en deux familles: dans la première les plaques s'écartent en s'accroissant par les bords et la distribution générale de la contrainte y est "horizontalement extensive", dans la seconde les plaques s'affrontent ou s'escamotent l'une sous l'autre, induisant alors une compression ou un cisaillement dans le sens horizontal. On notera que si une bande séismique a une distribution générale "extensive" ou "compressive" elle peut bien admettre localement et selon le moment, des zones restreintes à dis- tribution inverse, par exemple quand intervient la flexion. Utilisant l'échelle des durées communément employée, on pourra dire que la mosaïque figurée par les plaques admet un dessin quasiment fixe, et de cette façon il n'y a jamais de séismes en dehors des zones séismiques du moment. II n'en va pas de même si l'on considère les durées géologiques. car les mouvements des plaques, qui sont globaux, entraînent une évolution du dessin des limites. De cette manière une zone aujourd'hui séismique peut très bien perdre cette qualité demain et vice-versa.

On remarquera de plus, que la fréquence des séismes au sein d'une même zone est très variable. Ceci permet toujours avec utilisation de l'échelle des durées "humaines", de dire que certaines plages des zones séismiques sont pratiquement asismiques : tel le bassin parisien, par exemple, qui est pourtant dans la zone alpine au même titre que la bordure méditerranéenne, cependant beaucoup plus agitée.

Pour terminer ce préambule, jugé nécessaire pour un lecteur non géologue, je soulignerai encore le caractère temporel variable des contraintes naturelles, dont le développement se manifeste par crises. En l'état actuel, les causes déterminantes de ces moments d'agitation de la croûte terrestre sont très peu connues, et il est probable que leur distribution dans le temps n'est pas purement aléatoire.

\section{Variations de contrainte concomitantes des changements de niveau d'un lac réservoir.}

\subsection{Variations superficielles}

De l'importance en surface et en volume du lac constitué, découle la possibilité de pénétration profonde des effets engendrés. Cependant si petite soit la taille du réservoir, son remplissage entraîne inévitablement des changements de la distribution des contraintes dans les roches superficielles.

\section{action sur le fond de la cuvette}

Sur le fond d'une cuvette le poids de l'eau réalise un tassement d'autant plus important que la roche est plus compressible. C'est ainsi que par exemple dans les argiles pliocènes 
de la vallée du Rhône, intéressées par la retenue de St Vallier, pour une différence de charge d'une douzaine de mètres entre l'amont et l'aval du barrage, on a enregistré un tassement d'environ $4 \mathrm{~mm}$ [2]. Ce tassement s'annule vers les rives par effet de bord croissant avec la rigidité des roches considérées. Ces phénomènes qui sont délicats à séparer de ce qui revient en propreà la variation de pression interstitielle, ont été étudiés en détail pour plusieurs cuvettes de retenue [2].

\section{action sur les rives}

Au-delà de l'effet de bordure que je viens de rappeler, une autre variation s'instaure, dépendant cette fois de la perméabilité du terrain encadrant. Si les rives sont imperméables, l'eau de la cuvette ne pénètre pratiquement pas, et les conditions de pression sont inchangées. Au contraire dans les rives perméables le déjaugeage opéré, entraîne un gonflement mesurable. C'est ainsi qu'au Barrage de Foum el Gherza [3] (prés de Biskra, Algérie) la borne repère J située à environ $300 \mathrm{~m}$ à l'amont de l'ouvrage et à $30 \mathrm{~m}$ au-dessus des plus hautes eaux normales, a manifestè au remplissage (1952) un gonflement d'environ $1 \mathrm{~mm}$ par tranche d'eau de $10 \mathrm{~m}$. Ceci correspond à des variations du plan d'eau (positives ou négatives) au voisinage de la cote des plus hautes eaux normales. En effet, le phénomène a été dans un premier temps beaucoup plus complexe, car les calcaires maestritchiens très fissurés et peu cohérents ont été fortement désorganisés par le premier remplissage de sorte que la borne $\mathrm{J}$ a commencé par descendre anarchiquement de $17 \mathrm{~mm}$ avant de montrer la "respiration" régulière signalée. Pendant toutes ces variations l'appui calcaire rive gauche du barrage, grâce à l'efficacité de l'écran d'étanchéité réalisé par injections, annulant le déjaugeage, montrait une fixité remarquable à $0,1 \mathrm{~mm}$ près, ordre de grandeur de la précision des mesures.
Des observations aussi précises, mais faites uniquement en cotes d'altitude dans la zone du Chott ech Chergui (Hautes plaines algériennes) ont mis en évidence des tassements de l'ordre de $25 \mathrm{~mm}$ lorsqu'on a rabattu de $100 \mathrm{~m}$ la nappe aquifère contenue dans les calcaires jurassiques. II s'agit là d'un phénomène analogue mais non identique, pour lequel le terme de baisse de pression interstitielle remplacerait avantageusement celui de déjaugeage, la nappe étant en charge et non libre.

Plus loin encore dans ce sens on accède à la subsidence des zones pétrolifères oú la baisse de pression consécutive de l'exploitation est alors considérable, entraînant des tassements de même échelle.

\section{conséquences de ces variations superficielles}

Quoi qu'il en soit de ces mouvements du sol inhérents à la variation du niveau piézométrique, on conçoit aisément que les déformations conséquentes peuvent entraîner des ruptures, particulièrement dans les zones soumises au déjaugeage oủ la variation relative de pression est importante (de l'ordre de 10/25 au maximum à la surface). Ces ruptures sont donc nécessairement superficielles et se produisent dans les zones à dysharmonie de perméabilité, par exemple dans les calcaires karstifiés.

A Foum el Gherza que nous venons de citer, les calcaires maestritchiens proches d'une craie très fissurée se sont rompus sans brutalité et sans induire d'ondes élastiques (séismiques) directement perceptibles. Au Chott ech Chergui par contre où la nappe rabattue gisait dans les calcaires jurassiques largement karstifiés et très cohérents, plusieurs observateurs ont perçu des "explosions souterraines".

Si les roches s'y prêtent, à la fois par leur perméabilité et leur rigidité, le déjaugeage induit donc de très faibles secousses que l'on peut enregistrer et localiser avec un réseau de séis-

\section{Fig. 1 Carte géologique des gorges de l'oued Fodda (d'après Y. Gourinard 1952)}

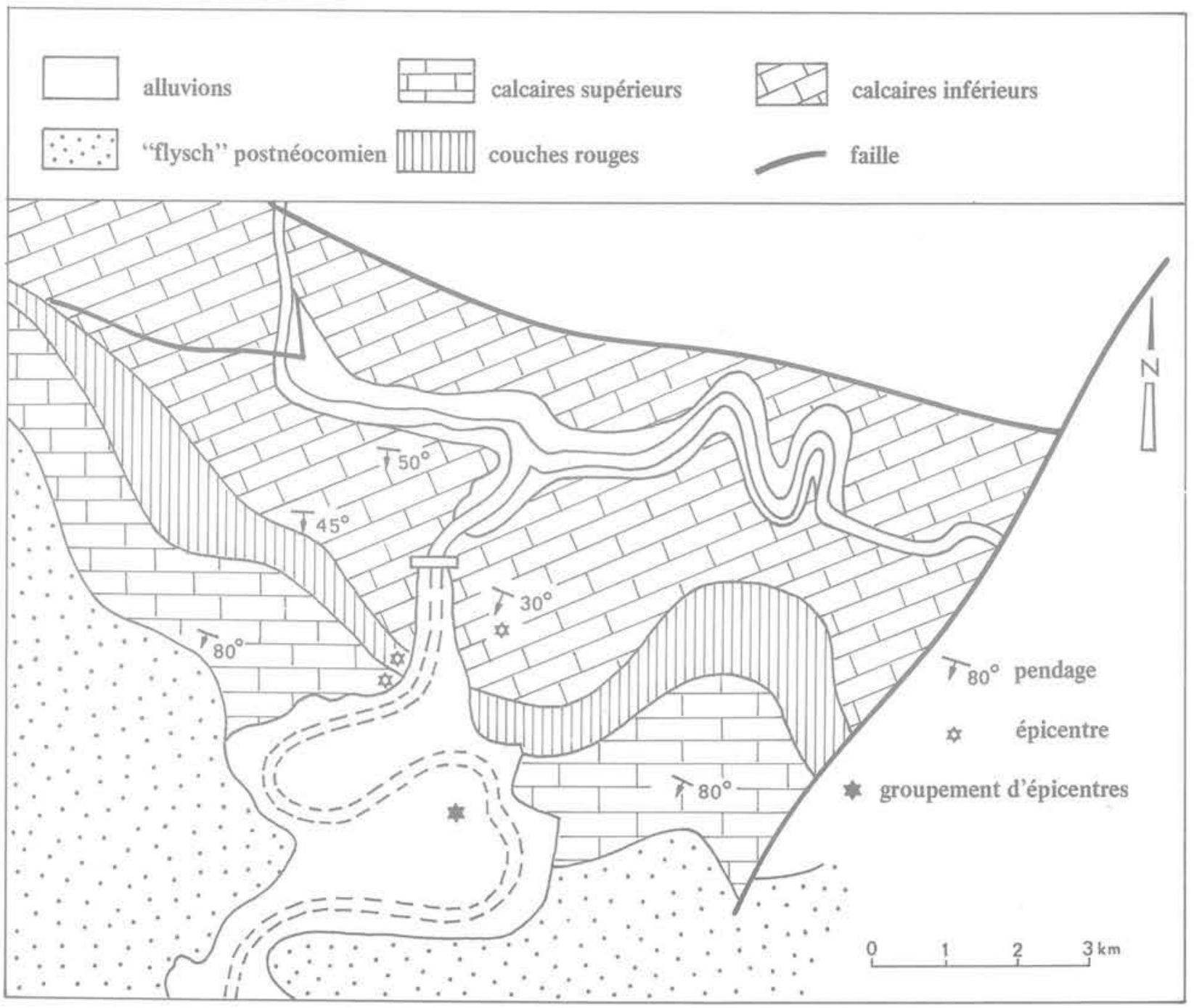


mographes adéquat.

C'est une expérience de ce genre qui a été poursuivie au barrage de Ste Croix sur le Verdon, oủ l'on a observé de nombreux séismes de très faible magnitude $(M<2)$ au premier remplissage [4]. Comme on aurait pu s'y attendre, les épicentres sont principalement localisés en rive gauche dans.les calcaires jurassiques très karstifiés. Toutefois si l'on admet l'hypothèse que je propose, les profondeurs calculées pour les foyers qui sont situés en moyenne à $3 \mathrm{~km}$ sous la surface, seraient entachées d'erreurs : en effet les calcaires intéressés totalisent au grand maximum $1000 \mathrm{~m}$ d'épaisseur.

\section{séismes au barrage d'Oued Fodda}

Ce barrage réalisé en Algérie vers 1930 ménageait une retenue de $225 \mathrm{hm}^{3}$ avec une profondeur d'eau maximale de $90 \mathrm{~m}$ (l'envasement a notablement réduit ce volume par la suite). Barrage poids, il s'appuie sur les calcaires jurassiques (voir fig. 1) de fort amont-pendage, cependant que la cuvette se développe sur des marnes et schistes crétacés, sauf dans le premier kilomètre à partir de l'ouvrage où les calcaires sont baignés.

Une structure géologique très complexe, remarquablement mise en évidence par Y. Gourinard [5], permet de supposer que l'os calcaire entaillé par les gorges, a enduré la submersion réalisée par les nappes crétacées, violemment charriées en direction du sud vers le milieu de l'époque tertiaire. De ce fait les calcaires en cause ont été fortement malmenés et l'on peut supposer qu'ils portent encore aujourd'huil les traces de ce traumatisme, sous la forme de contraintes résiduelles. Dans une situation tectonique analogue, les schistes du barrage de la Bou Namoussa (près d'Annaba,
Algérie) dans lesquels on avait pratiqué en 1952 une galerie de reconnaissance rive droite, avaient permis de mesurer un pareil défaut de relaxation, avec grand bénéfice pour la contrainte horizontale de compression.

Or, lorsqu'à l'Oued Fodda on a réalisé la mise en eau, toute une série de secousses ont été ressenties, dont certaines à $6 \mathrm{~km}$ de distance. Un dispositif d'observation et de mesure, admirablement agencé pour l'époque (1932) à l'aide d'appareils de prospection transformés pour les besoins de la cause, a localisé les épicentres exclusivement dans les calcaires noyés par la retenue, les foyers étant à moins de $300 \mathrm{~m}$ de profondeur [5]. La plus grande concentration de foyers correspondait à une zone très malmenée par la tectonique, logiquement la plus karstifiée (voir fig. 1).

On doit se demander pourquoi le simple déjaugeage a pu provoquer des ruptures aussi brutales, induisant des secousses non destructrices, mais perçues sous un jour inquiètant par les forestiers voisins... Une réponse peut être proposée grâce à l'état de contrainte que l'on vient de supposer préexistant dans les calcaires d'Oued Fodda. En effet. la sous-pression inhérente au déjaugeage s'est ajoutée à la contrainte naturelle dans les zones superficielles où se sont produites les ruptures, cependant qu'elles se retranchait en-dessous, là oủ était reporté le poids de l'eau, consolidant ainsi les zones oủ celles-ci ne pénétrait pas. Tel est en tout cas le mécanisme que je propose; j'ai d'ailleurs déjả fait appel à une hypothèse semblable, mais à une autre échelle, pour justifier de ce qui peut se passer beaucoup plus profondément [6].

Avant de quitter le domaine superficiel, il paraît peut-être souhaitable de dire quelques mots de très faibles secousses séismiques syndromiques de la catastrophe du Vajont (Italie).

Fig. 2 Pluie et fréquence des secousses au réservoir du Vajont (d'après Galanopoulos (1967) et EDF (1965)

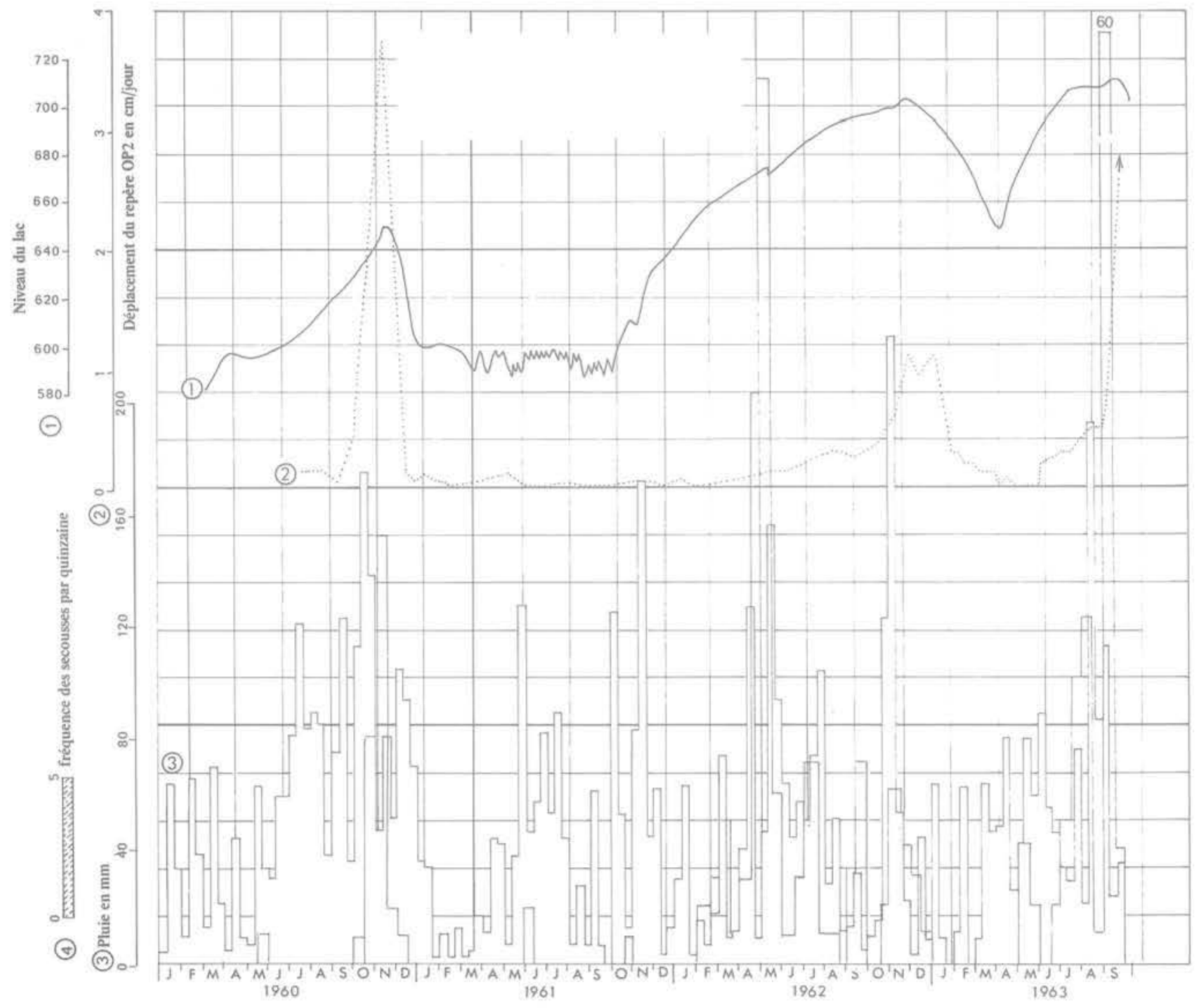




\section{séismes du Vajont}

On sait qu'un gigantesque glissement-éboulement est "tombé" dans la retenue du Vajont en 1963. Or de trés nombreuses petites secousses séismiques se sont manifestées pendant la mise en eau, réalisée par paliers récurrents et recouvrants de Mai 1960 à Septembre 1963 date de la catastrophe. On a mis ces phénomènes en relation avec le remplissage du lac [7] et l'on peut supposer par là que le déjaugeage est en cause (par le biais des ruptures à l'origine du mouvement). De fait une petite partie du pied du glissement a été soumise à ce genre de sous-pression. Cependant si l'on superpose la fréquence des secousses à la hauteur de pluie (v. fig. 2), on constate une corrélation du même style, et c'est logique car d'une part on remplit le lac avec la pluie environnante et non lointaine, et d'autre part les chances de ciel bleu localisé aux seules zones de glissement sont pratiquement nulles. Ceci, appuyé par la détermination de nombreux épicentres sur le site origine du glissement, permet de supposer que les précipitations abondantes accélèraient le mouvement des terrains, donc la fréquence des ruptures, et des petites secousses séismiques conséquentes. II faut également supposer que la décharge au pied, liée au déjaugeage, facilitait les ruptures. Postérieurement à la catastrophe d'ailleurs, l'équilibre des terrains déplacés constamment remis en cause et par les précipitations atmosphériques abondantes et par les changements de niveau du lac, a été l'origine pendant plusieurs années de nombreuses très petites secousses séismiques [8].

Ne disposant pas de toute la documentation nécessaire, je prie les auteurs qui ont certainement déjà émis ces hypothèses, de bien vouloir m'excuser et de transformer mes assertions en citations à leur bénéfice.

\subsection{Variations profondes}

Quelle que soit l'importance du lac réservoir et quelles que soient les hypothèses faites sur les qualités de la croūte terrestre, les calculs faits à la suite des frères Gough [9] par plusieurs chercheurs, ne peuvent assurément mettre en évidence en profondeur des variations de pression supérieures à la pression réalisée par l'eau en surface. Ainsi la variation relative en profondeur devient vite très faible, et sans commune mesure avec les contraintes naturelles capables de rompre la croûte terrestre.
Ce fait a été bien souligné par le groupe de travail Unesco réuni à Paris en 1970, et je l'ai personnellement pris à mon compte en 1971 [6] sous l'angle des théories géologiques actuelles. Se reportant en effet au préambule de la présente communication, et sachant que le lac de Kariba sur le Zambèze est installé sur un fossé d'effondrement naturel, constituant typiquement une zone en extension, on ne s'étonnera pas du séisme violent $(M>6)$ qui a secoué cette région au remplissage. Est-il besoin de rappeler une fois encore, que la distribution des contraintes préexistantes étant "extensive" le poids de l'eau vient s'ajouter aux efforts naturels... que l'on est obligé de supposer avoir approché la limite de rupture à ce moment là, ce qui ne libère en rien le remplissage du lac de sa responsabilité. Le mécanisme calculé au foyer [10] pour Kariba s'inscrit très bien dans la théorie puisqu'il correspond à une extension horizontale, provoquant l'effondrement du compartiment portant le lac.

\section{Cas du barrage de Granval}

C'est ả une échelle beaucoup moins redoutable que l'exemple français du barrage achevé sur la Truyère en 1959 nous offre un mécanisme parfaitement similaire. La carte géologique correspondante (voir fig. 3) localise la retenue sur une des "Limagnes" du Massif Central français. Les Limagnes s'identifient à des fossés d'effondrement qui ont commencé de jouer au début de l'époque tertiaire (Oligocène). Le jeu de failles associées, grossièrement orienté NW-SE n'est pas partout aussi nettement matérialisé, mais la direction des lignes de drainage impose l'existence réelle des accidents qui sont seulement supposés sur la figure 3 . Là encore les fossés d'effondrement sont nés par le fait de l'extension horizontale naturelle, déjà brutalement manifestée avant la construction du barrage $(1861,1862,1889$, 1918 ) et qui devrait être proche de la rupture en 1963. Comme on peut le déduire des propositions précédentes, le remplissage* a été suivi de secousses séismiques d'intensité macroséismique V. Les trois secousses en cause n'ont pratiquement pas fait de dommages, mais n'ont pas laissé de tourmenter les occupants de l'usine à l'époque.

Bien que la méthode de détermination des épicentres utilisée ne garantisse leur précision qu'à quelques kilomètres

- pour toutes les données temporelles précises le lecteur voudra bien se reporter au rapport $\mathrm{R} 37, \mathrm{Q} 61$. XIII' C.I.G.B. Paris.

\section{Fig. 3 Carte géologique de la zone du barrage de Granval (d'après EDF 1979)}

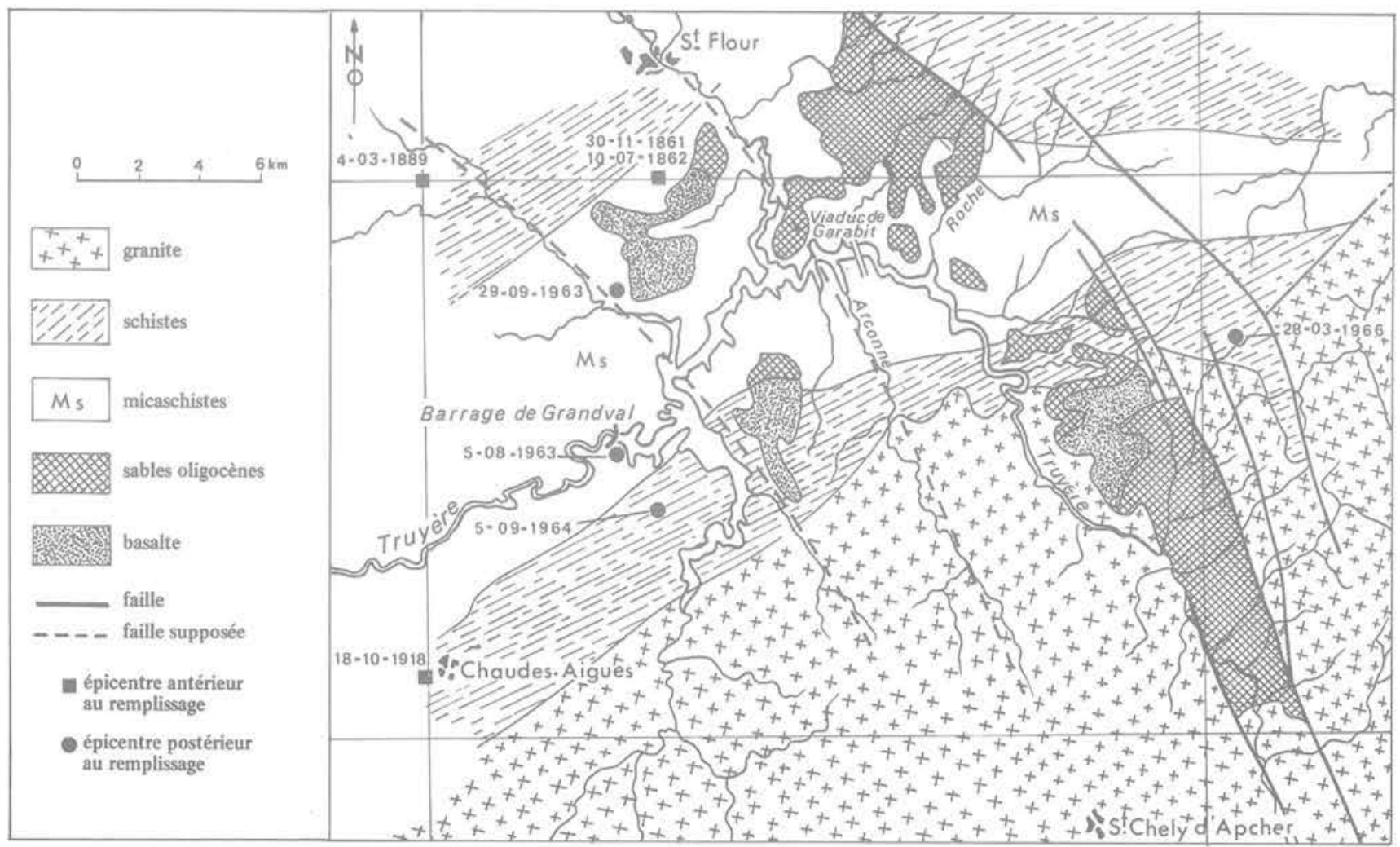


près, leur groupement satisfait correctement à l'hypothèse proposée. Cet exemple fait à nouveau constater qu'une faible charge réalisée par la retenue $\left(270 \mathrm{hm}^{3}, 80 \mathrm{~m}\right.$ de hauteur d'eau maximale) parfaitement filiforme en comparaison de celle de Kariba, s'est ajoutée à une contrainte naturelle "extensive" rendue très proche de la rupture. On notera que les compartiments intéressés par le réservoir semblaient à l'époque avoir atteint un certain équilibre car deux ans plus tard c'est nettement plus à l'Est, mais toujours dans une "Limagne" que les contraintes naturelles se sont libérées. L'exemple de Granval peut paraître le plus démonstratif des cas français en vérité très peu nombreux. On aurait cependant tort de négliger celui de Monteynard " situé dans les Alpes, près de leur bordure occidentale. Le lac de retenue correspondant bénéficie de conditions structurales analogues puisqu'il recouvre sur une part importante de sa longueur une faille bordière du Vercors, effondré à l'Ouest. Toutefois le mécanisme d'extension E-W n'est pas ici aussi évident que dans les "Limagnes".

\section{barrage d'Eguzon}

Continuant de descendrel'échelle des magnitudes, j'examinerai maintenant ce qui s'est passé dans une autre région du Massif Central français, près de sa bordure septentrionale. Ces marges, notamment entre la Creuse et le Cher (voir fig 4) sont affectées de mouvements en "touches de piano" ennoyant les terrains cristallins vers le Nord.

Se conformant aux règles de l'évolution géomorphologique la Creuse suit d'assez près une des failles de ce réseau. Le petit barrage d'Eguzon ( $55 \mathrm{hm}^{3}, 61 \mathrm{~m}$ de hauteur en crête) a été construit sur cette rivière en 1925. Un but de recherche poursuivi dans une autre direction que celle des barrages réservoirs a nécessité l'établissement de plusieurs stations séismiques modernes et très "performantes" (voir fig. 4). Celles-ci ont enregistré au printemps 1977 plusieurs séries de petites secousses séismiques ( $\max . \mathrm{M} \simeq 3,4$ ) dont certaines ont été nettement perçues par des observateurs humains en provoquant la chute de quelques plattras. L'exploitation compétente des enregistrements [11] met en évidence un essaim de séismes peu profonds, pour lesquels le mécanisme focal calculé est en excellent accord géométrique avec la direction et le pendage de la faille guide de la Creuse, ainsi qu'avec l'effondrement du compartiment sur lequel est installée la maigre retenue d'Eguzon.

II faut donc constater qu'une crise séismique a tourmenté la région en 1977 et que des ruptures conséquentes d'une extension, se sont préférentiellement produites là où la faible charge induite pourtant depuis un demi siècle par ce petit lac, avait pu s'additionner aux contraintes naturelles.

- voir le rapport R 37, Q 61, XIII* C.I.G.B. Paris.

Fig. 4 Essaim de séismes de la zone d'Eguzon

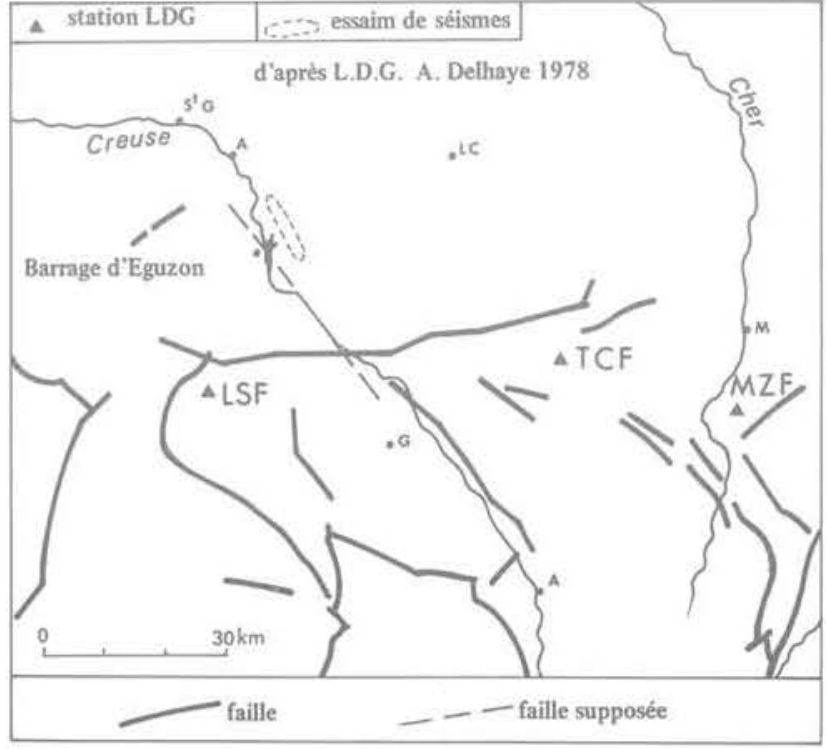

\section{Conclusions}

Descendant l'échelle logarithmique des magnitudes nous sommes arrivés jusqu'au repère "Eguzon" où l'ordre de grandeur des énergies mises en jeu paraît à peu près comparable à celui constaté á l'Oued Fodda. Or si ce dernier lieu a abrité des phénomènes rangés sans aucune hésitation dans le domaine tout à fait superficiel, en revanche, c'est à queiques kilomètres de profondeur que se sont amorcées les faibles ruptures d'Eguzon.

Dans ces conditions on est tenté de penser qu'il n'y a pas de différences fondamentales de genèse, entre tous les phénomènes vibratoires déclenchés dans le matériau terrestre par le remplissage de toutes les retenues. En effet, depuis les craquements presque imperceptibles, nés dans la digue elle-même, dont les constituants prennent leur place avec plus ou moins de gêne, jusqu'aux séismes destructeurs engendrés en pleine croute terrestre, il s'agit toujours d'ondes que l'on peut qualifier d'élastiques syndromiques de ruptures, ruptures centimétriques en surface, failles kilométriques voire multikilométriques, dans la profondeur. En outre il est possible d'imaginer une belle continuité que pourraient assurer les microruptures omniprésentes, et que les séismologues modernes associent maintenant à la dilatance, dans l'étude des phénoménes prémonitoires des séismes destructeurs et meurtriers.

Pour respecter le cadre humaniste au sein duquel devraient s'inscrire toutes les préoccupations des ingénieurs, séismologues et géologues, il n'est pas permis de quitter la discussion par le seul côté spéculatif; c'est pourquoi je tenterai au moins d'entr'ouvrir l'autre battant de cette seule et même porte donnant sur la recherche scientifique.

Essayant donc de faire le bilan de ce que la géologie apporte dans la résolution du problème posé par la séismicité induite par les lacs réservoirs, on reconnaîtra sans peine qu'il n'est pas négatif. L'hypothèse présentée, séduisante par sa simplicité, dispose déjà d'un certain nombre de faits concourants ayant valeur d'argument. L'auteur souhaiterait que les exemples dont disposent les autres pays aménageant des lacs de retenue, soient examinés par les spécialistes répondants aussi bien sous le jour de la qualité des contraintes préexistantes que du mécanisme focal. Si ce dernier peut être assez bien cerné par le calcul, il ne vient malheureusement qu'après coup avec sa pâle figure de confirmation ! Restent donc les contraintes préexistantes avec leur distribution "extensive" ou "compressive", et c'est dans ce domaine qu'une carte géologique précise, mais surtout située dans son contexte tectonique général connu par l'analyse structurale, doit être capable d'indiquer par la voie du géologue averti, le sens dans lequel risquent d'évoluer les contraintes naturelles profondes... sous la dépendance desquelles nous resterons sans doute encore longtemps.

Ainsi, laissant pour le futur la lutte antiséismique active, le géologue pourra indiquer l'influence éventuelle d'une retenue, cependant que le séismologue supputera les chances de cet évènement en fonction du passé séismique de la région, quant à l'ingénieur il devra prendre les dispositions nécessaires pour que l'évènement possiblement redouté, ait le moins de conséquence possible aussi bien sur le barrage que sur les œuvres humaines environnantes. On notera que faute de concertation cette action perdrait beaucoup de l'efficacité recherchée.

\section{Références Bibliographiques}

[1] J. COULOMB - La constitution physique de la terre. Albin Michel Paris 1952.

[2] P. SAVEY - Gonflement et tassement d'un substratum argileux utilisé pour la fondation de grands ouvrages. Annales de I'ITBTP. Supplément au n 334 Paris 1975.

[3] R. ARIS - Les travaux d'ètanchement des terrains au barrage de Foum el Gherza. CIGB. Q 16, R 43 Paris 1955. 
[4] J.N. PLICHON, P. GEVIN, HOANG TRONG PHO, P. LONDE, P. PETITEVILLE - XIII ${ }^{\circ}$ Congrès International des Grands Barrages, Q 61, R 37, Paris 1979.

[5] Y. GOURINARD, J. THEVENIN - Le barrage de l'Oued Fodda. XIX Congrès géologique International. Alger 1952.

[6] P. GEVIN - Séismes et barrages. C.R. Acad. Sci. Paris, t. 276, 2.4.73.

[7] GALANOPOULOS A.G. - Influence des variations de niveau du lac de Marathon sur l'activité séismique locale de la zone du bassin Attique. Annales géologiques des Pays Hélléniques. Athènes 1967.
[8] CALOI P. - Ann. Geofis. No 19, pp. 1-74, Roma 1970. [9] GOUGH D.I, and GOUGH W.I. - Stress and deflection in the lithosphere near lake Kariba. Geophys. J. $n^{\circ} 21$, London 1970.

[10] GUPTA H.K., RASTOGI B.K, and NASAIN H. Some discriminatory characteristics of earthquakes near the Kariba, Kremasta and Koyna artificial lakes. Bull. seismol. Soc. Am. n62, pp. 47-61.

[11] A. DELHAYE, N. LACHAIZE, J.P. SANTOIRE - La séismicité 1977 dans la bordure $N$ du Massif central et ses implications tectoniques. $6^{\circ}$ Réunion annuelle des Sciences de la Terre Paris 1978. 